\title{
Formation of Dislocation Structure in LiF Crystals Irradiated with Swift Heavy Ions under Oblique Incidence
}

\author{
A. Russakova*, A. Akilbekov, A. Dauletbekova and M. Baizhumanov \\ L.N. Gumilyov Eurasian National University, Astana, 5 Munaitpasov Str., 010008, Kazakhstan
}

\begin{abstract}
The structural modifications of $\mathrm{LiF}$ irradiated with swift heavy ions under oblique angles have been investigated using AFM, SEM, chemical etching, nanoindentation and optical absorption spectroscopy. LiF crystals were irradiated under incidence angles of 30 and 70 degrees with $2.2 \mathrm{GeV} \mathrm{Au}$ (fluence $5 \times 10^{11}$ ions cm ${ }^{-2}$ ) and $150 \mathrm{MeV}$ $\mathrm{Kr}$ ions (fluence $10^{12}-10^{14}$ ions $\mathrm{cm}^{-2}$ ). Structural study on sample cross-sections shows that two damage regions, (1) nanostructured zone and (2) dislocation rich zone, which are typical for irradiations at normal incidence, appear also in samples irradiated under oblique angles. However in the latter case a more complex structure is formed that leads to stronger ion-induced hardening.
\end{abstract}

DOI: $10.12693 /$ APhysPolA.125.1257

PACS: $42.88 .+\mathrm{h}, 61.80 . \mathrm{Jh}, 71.20 . \mathrm{Dg}$

\section{Introduction}

The modifications of the structure of materials on the micro- and nanometer scale and an improvement of their properties by irradiation with swift heavy ions (SHI) are of importance on both the fundamental and technological standpoint. High fluence irradiation of ionic crystals with SHI leads to severe structural modifications including formation of latent tracks and surface and bulk nanostructures [1-3]. A significant role in the self-organized nanostructuring is played by stress fields of extended defects (such as tracks and dislocations) as well as crystallographic orientation. The latter is of special importance for ionic crystals which maintain crystallinity even under high-fluence irradiation. In the majority of studies the ion beam has been oriented normal to the crystal surface and tracks propagate along particular crystallographic direction. Peculiarities of track damage were observed at irradiation of crystal surfaces under grazing incidence of ion beam [4].

In this study the structural modifications in the bulk of $\mathrm{LiF}$ crystals irradiated under oblique incidence of ions has been investigated using atomic force microscopy (AFM), scanning electron microscopy (SEM), chemical etching, nanoindentation, and optical absorption spectroscopy. Irradiation was performed with $2.2 \mathrm{GeV}$ Au ions, which in $\mathrm{LiF}$ produce complex tracks consisting of core region containing small defect aggregates and a surrounding halo region where mostly single defects are created [1]. Such tracks similar to dislocations exhibit stress fields and can be revealed by selective chemical etching. In this study also $150 \mathrm{MeV} \mathrm{Kr}$ ions have been used which are attractive in a way that the threshold of energy loss for the core damage is surpassed only in a certain part of the ion range. As a result, two distinctively different in structure regions on the etched profile surfaces are observed [3].

\footnotetext{
*corresponding author; e-mail: arussakova@gmail.com
}

\section{Experimental}

Irradiations were performed on nominally pure $\mathrm{LiF}$ crystals (Korth Kristalle, Germany) at the UNILAC linear accelerator of the GSI Darmstadt with $2.2 \mathrm{GeV}$ $\mathrm{Au}$ ions at dose of $5 \times 10^{11}$ ions $/ \mathrm{cm}^{2}$ under normal and oblique (30 degrees) incidence to the (100) cleavage face and at the cyclotron DC-60 (Astana, Kazakhstan) with $150 \mathrm{MeV}{ }^{84} \mathrm{Kr}^{+14}$ ions in the fluence range $10^{11}-10^{14}$ ions $/ \mathrm{cm}^{2}$ under incidence angles of 30 and 70 degrees. All irradiations were performed at room temperature. Ion tracks and dislocations were revealed using chemical etching.

\section{Discussion}

\subsection{Dependence of the thickness of irradiated layer on angle of incidence}

The thickness of the irradiated layer in the case of normally and under oblique incidence irradiated samples was estimated from optical microscopy of etched profile surfaces. The irradiated layer can be observed as striated structure oriented along the direction of the ion beam. For the normally irradiated samples the thickness of the irradiated layer coincided with the ion range $R$, which was calculated using the SRIM code [5]. For the samples irradiated under oblique angles the damaged layer was thinner. Its thickness is in agreement with the estimates performed from the geometrical considerations as $h=R \sin \alpha$, where $\alpha$ is the angle of incidence (Fig. 1). The result confirms the independence of ion range of the ion incidence angle. Besides, samples which are inclined against ion beam receive correspondingly lower irradiated dose due to projection of exposure over larger area.

The results of optical absorption spectroscopy for simultaneously irradiated samples under normal and oblique incidences are also in agreement with this model. Weaker coloration was found in samples irradiated under lower angles of incidence (Fig. 2). The lower optical density is caused not only by a thinner irradiated layer but 


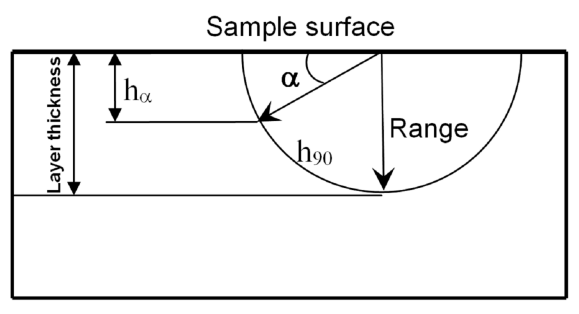

Fig. 1. The thickness of the irradiated layer in dependence on the incidence angle of ions (scheme).

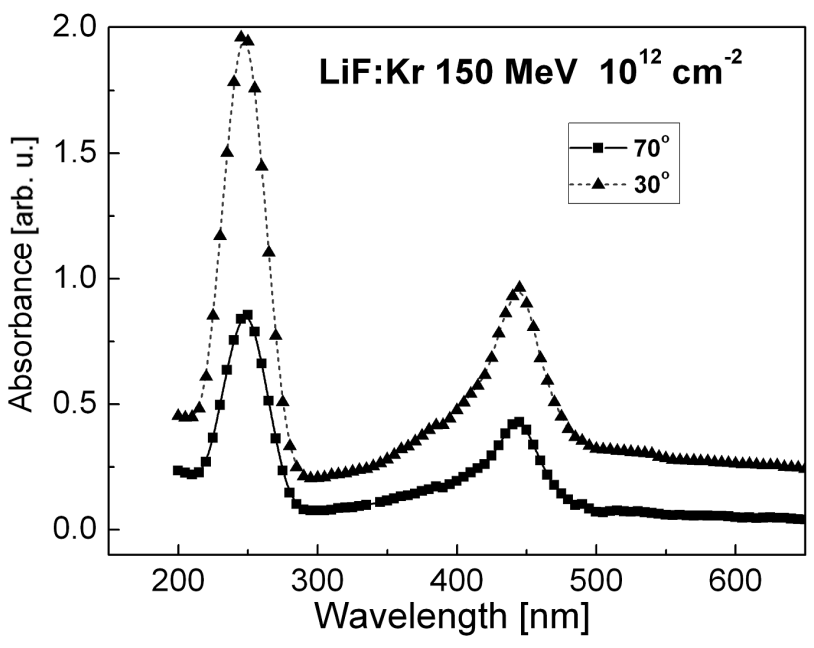

Fig. 2. Absorption spectra for $\mathrm{LiF}$ crystals irradiated with $150 \mathrm{MeV} \mathrm{Kr}$ ions at $10^{12}$ ions $/ \mathrm{cm}^{2}$ under $70^{\circ}$ and $30^{\circ}$ incidence.

also by a larger surface area corresponding to a lower dose. Spectra for all samples were similar and showed $F, F_{2}$, and $F_{n}$ absorption bands typical for high fluence irradiations $[1,3]$.

\subsection{Structural study on the irradiated surface}

On the surfaces irradiated with $\mathrm{Au}$ and Kr projectiles etchable tracks were observed (Fig. 3a). Such result was expected because in both cases the energy loss for the ions surpasses the critical threshold of $10 \mathrm{keV} / \mathrm{nm}$ for core damage and track etching [1]. For the samples irradiated under oblique angle also the etch pits of tracks were tilted (Fig. 3a, inset).

\subsection{Structural study on the cross-sections of samples}

The structural study was performed on cross-sections prepared by cleaving perpendicular to irradiated (001) face. At all irradiations under oblique angles the total thickness of irradiated layer was lower than the ion range and varied with the incidence angle as described in Sect. 3.1.

Our previous studies showed that at high fluence irradiation with $\mathrm{Kr}$ ions under normal angle, two regions exhibiting different types of structural features appear: (1) the nanostructured zone which can be characterized

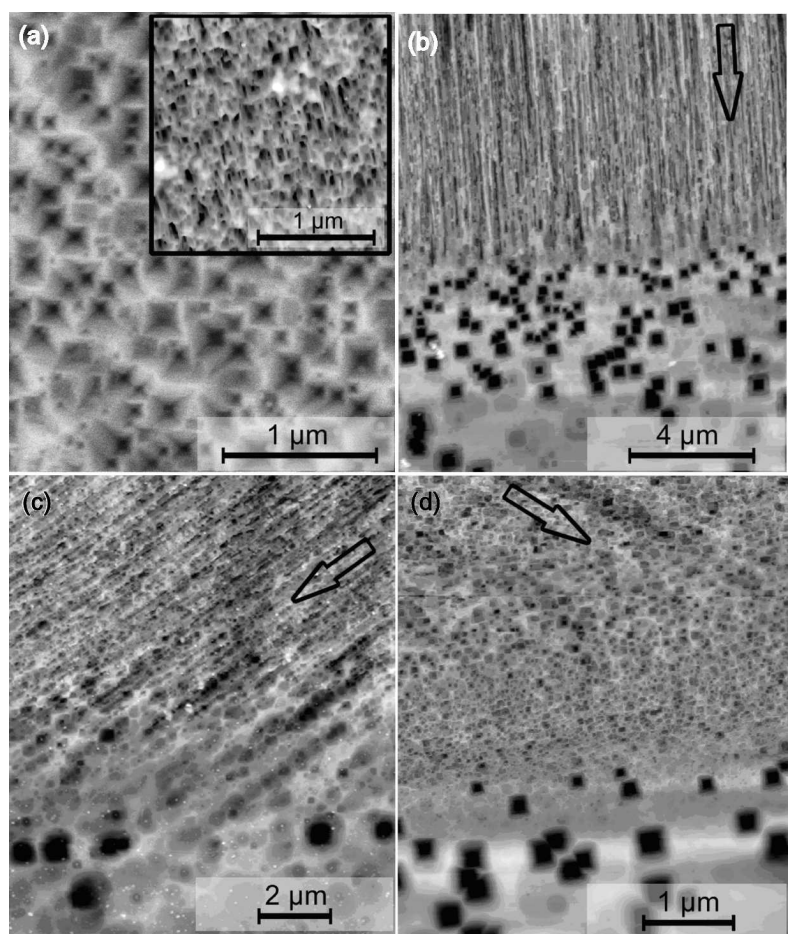

Fig. 3. Damage structures created under normal and oblique incidence of ions (AFM and SEM images): (a) etching of Au tracks on normally irradiated surface; fluence $5 \times 10^{11}$ ions $\mathrm{cm}^{-2}$. Inset shows etched tracks under $30^{\circ}$ incidence; (b) profile surface, normal irradiation with $\mathrm{Kr}$ ions at $10^{12}$ ions $/ \mathrm{cm}^{2}$; (c) profile surface, $\mathrm{Au}$ ions under $30^{\circ}$ incidence, fluence $2.5 \times 10^{11}$ ions $\mathrm{cm}^{-2}$; (d) profile surface, irradiation with $\mathrm{Kr}$ ions under $30^{\circ}$ incidence at $10^{12}$ ions $/ \mathrm{cm}^{2}$. Arrows show the direction of irradiation.

as mosaic type structure with small subgrains and (2) the zone containing numerous ion-induced dislocations (Fig. 3b) [2,3]. The two structural types were obtained also for all investigated incidence angles of Kr ions. The proportion of their extension was approximately the same as for irradiations under normal angle. Figure 3d shows the structure formed on profile surface of sample irradiated with $\mathrm{Kr}$ ions at the angle of $30^{\circ}$.

In the case of $\mathrm{Au}$ ions, the energy loss of which in $\mathrm{LiF}$ strongly exceeds that for $\mathrm{Kr}$ ions, the high fluence irradiation under normal and oblique angles in a dominant part of the irradiation zone creates a typical for nanostructures striated relief oriented in the direction of incoming ions (Fig. 3c).

\subsection{Nanoindentation measurements}

Nanoindentation testing on normally irradiated surface revealed substantial increase of hardness in agreement with our earlier results $[2,3]$. Nevertheless in samples irradiated under oblique angle the hardening effect was stronger despite of the lower received irradiation dose (Fig. 4). Taking into account that hardness is sensitive to defect aggregates, the result points to certain difference 
in their ordering in dependence of irradiation angle. In agreement with the scheme (Fig. 1), the thickness of the hardened zone at oblique irradiation $\left(30^{\circ}\right)$ is about half of that for perpendicularly irradiated sample.

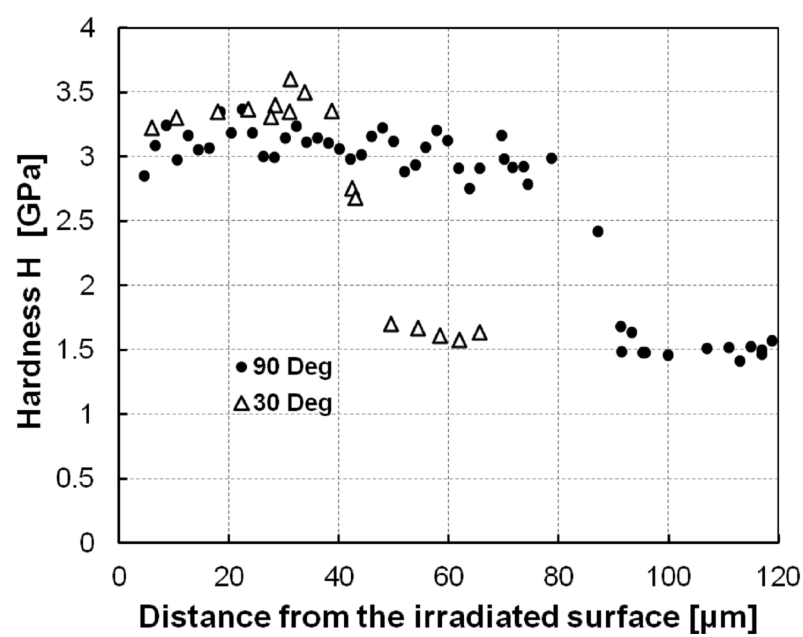

Fig. 4. Hardness vs. indentation depth on profile surface of samples irradiated with $\mathrm{Au}$ ions under $90^{\circ}$ (solid symbols) and $30^{\circ}$ (open symbols) incidence angle. The fluence was $5 \times 10^{11}$ and $2.5 \times 10^{11}$ ions $\mathrm{cm}^{-2}$, correspondingly.

\section{Conclusion}

The thickness of irradiated layer varies with the incidence angle of ions. Maximum thickness which equals to the calculated ion range $R$ is observed at irradiation perpendicularly to the (001) surface of a sample. The thickness of the irradiated layer decreases with decrease of the incidence angle as $h=R \sin \alpha$ in agreement with geometrical considerations. The results confirm that the ion range is independent of the incidence angle of ions.

Structural study shows that two zones of structural damage - nanostructured zone and dislocation rich zone, which are typical for irradiations at normal incidence [2, 3], appear also in samples irradiated under oblique angles. The extension of these zones depends on energy loss of projectiles.

The irradiated layer for all investigated incidence angles exhibits improved hardness that gives evidence for formation of defect aggregates, such as dislocations and other extended defects.

\section{References}

[1] C. Trautmann, K. Schwartz, J.M. Costantini, T. Steckenreiter, M. Toulemonde, Nucl. Instrum. Methods Phys. Res. B 146, 367 (1998).

[2] J. Maniks, I. Manika, R. Zabels, R. Grants, E. Tamanis, K. Schwartz, Nucl. Instrum. Methods Phys. Res. B 282, 81 (2012).

[3] A. Dauletbekova, J. Maniks, I. Manika, R. Zabels, A.T. Aklibekov, M.V. Zdorovets, Y. Bikert, K. Schwartz, Nucl. Instrum. Methods Phys. Res. B 286, (2012).

[4] I.V. Vorobyova, Nucl. Instrum. Methods Phys Res. B 198, (2002).

[5] J.F. Ziegler, P. Biersack, U. Littmark, The Stopping and Range of Ions in Matter, Pergamon Press, New York 1985. 